\title{
Avaliação ultrassonográfica e mensurações das glândulas adrenais em primatas não humanos neotropicais: mico-de- -cheiro (Saimiri sciureus), macaco-da-noite (Aotus azarae infulatus) e bugio-ruivo (Alouatta guariba clamitans) ${ }^{1}$
}

\author{
Andressa C. Souza르, Rogério R. Lange ${ }^{2}$, Andreise C. Przydzimirski², Paulo H.G. \\ Castro $^{3}$, Ana Pascoli ${ }^{4}$, Júlio César S. Júnior ${ }^{4}$ e Tilde R. Froes ${ }^{2 *}$
}

\begin{abstract}
Souza A.C., Lange R.R., Przydzimirski A.C., Castro P.H.G., Pascoli A., Junior J.C.S. \& Froes T.R. 2014. [Adrenal glands measurements and ultrasound evaluation in nonhuman primates: squirrel monkey (Saimiri sciureus), owl monkey (Aotus azarae infulatus) and howler monkey (Alouatta guariba clamitans).] Avaliação ultrassonográfica e mensurações das glândulas adrenais em primatas não humanos neotropicais: mico-de-cheiro (Saimiri sciureus), macaco-da-noite (Aotus azarae infulatus) e bugio-ruivo (Alouatta guariba clamitans). Pesquisa Veterinária Brasileira 34(9):903-910. Departamento de Medicina Veterinária, Universidade Federal do Paraná, Rua dos Funcionários 1540, Curitiba, PR 80035-050, Brazil. E-mail: froestilde@gmail.com

The adrenal glands have endocrine functions related to multiple vital functions and are closely related to the animal's ability to adapt to stress. The ultrasound is the diagnostic method of choice for evaluation of glands in different species. Considering the scarce literature, one may question whether the adrenal disorders in nonhuman primates are uncommon or underdiagnosed, and a hypothesis exists that this fact is determined by the lack of parameters. The goal is to describe the sonographic features of the adrenal glands for three species of nonhuman primates kept in captivity: squirrel monkey (Saimiri sciureus), owl monkey (Aotus azarae infulatus) and howler monkey (Alouatta guariba clamitans). It is concluded that it is possible to identify the adrenal glands by ultrasound, and the reference standards have been established successfully for the species in question. It is noteworthy that the adaptation of animals in many stressful environments is often accompanied by a hypertrophy of the adrenal glands, so one should take into account that the measurements performed in this study were established in captive animals.
\end{abstract}

INDEX TERMS: Adrenal glands, squirrel monkey, Saimiri sciureus, owl monkey, Aotus azarae infulatus, howler monkey, Alouatta guariba clamitans, Cushing, hyperadrenocorticism, hormones, ultrasound.

RESUMO.- As glândulas adrenais possuem funções endócrinas relacionadas a múltiplas funções vitais, estando intimamente relacionadas à capacidade do animal em se

\footnotetext{
${ }^{1}$ Recebido em 20 de março de 2014.

Aceito para publicação em 2 de julho de 2014.

2 Departamento de Medicina Veterinária, Universidade Federal do Paraná (UFPR), Rua dos Funcionários 1540, Curitiba, PR 80035-050, Brasil. *Autor para correspondência: froestilde@gmail.com

${ }^{3}$ Médico Veterinário, Secretaria de Vigilância em Saúde (SVS), Centro Nacional de Primatas (Cenp), Rodov. BR-316 Km 7, Ananindeua, PA 67030-000, Brasil.

${ }^{4}$ Departamento de Medicina Veterinária, Fundação Universidade Regional de Blumenal (FURB), Rua Antônio da Veiga 140, Blumenau, SC 89012900, Brasil.
}

adaptar ao estresse. 0 exame ultrassonográfico é o método diagnóstico de escolha para avaliação das glândulas em diferentes espécies. Considerando a escassa literatura, questiona-se se as doenças adrenais em primatas não humanos são incomuns ou subdiagnosticadas, havendo a hipótese desse fato ser determinado pela falta de parâmetros. Objetivou-se descrever as características ultrassonográficas das glândulas adrenais para três espécies de primatas não humanos mantidas em cativeiro: Saimiri sciureus (mico-de-cheiro), Aotus azarae infulatus (macaco-da-noite) e Alouatta guariba clamitans (bugio-ruivo). Conclui-se que é possível a identificação das glândulas adrenais por meio de exame ultrassonográfico, sendo que os padrões de referência foram estabelecidos com sucesso para as espécies em 
questão. Ressalta-se que a adequação de animais em ambientes estressantes é frequentemente acompanhada por uma hipertrofia das glândulas adrenais, portanto deve-se levar em consideração que as mensurações realizadas nesse estudo foram estabelecidas em animais de cativeiro.

TERMOS DE INDEXAÇÃO: Glândulas adrenais, mico-de-cheiro, Saimiri sciureus, macaco-da-noite, Aotus azarae infulatus, bugio-ruivo, Alouatta guariba clamitans, Cushing, hiperadrenocorticismo, hormônios, ultrassonografia.

\section{INTRODUÇÃO}

As glândulas adrenais são estruturas retroperitoneais pares, localizadas na região craniodorsal, medialmente aos rins em cães e gatos, sendo que a glândula esquerda encontra-se adjacente à aorta e a direita à veia cava caudal (Graham 2008, Mogicato et al. 2011). Em seres humanos estão localizadas também retroperitonealmente, porém no plano superior dos polos dos rins, denominadas de glândulas suprarrenais por esse motivo, apesar da adrenal esquerda estar posicionada ínfero-medialmente em relação ao polo cranial do rim esquerdo (Baroni et al. 2002).

Cada glândula possui uma camada cortical e uma medular, as quais produzem diferentes tipos de hormônios, sendo predominante o cortisol (Cunningham 2004). Suas funções endócrinas estão relacionadas com múltiplas funções vitais do organismo e desse modo qualquer alteração funcional pode ocasionar variados sinais clínicos nos animais (Graham 2008). 0 conjunto de hormônios secretados por essas glândulas está intimamente relacionado à capacidade do animal em se adaptar às condições adversas do meio ambiente, ou seja, ao estresse (Cunningham 2004).

0 exame ultrassonográfico é o método diagnóstico de escolha para avaliação das glândulas adrenais em diferentes espécies, possibilitando a visualização do parênquima e eventualmente de nódulos ou massas, permitindo também a realização de mensurações (Barthez et al. 1998, Baroni et al. 2002, Graham 2008). Para isto, se faz necessário o uso de equipamentos de ultrassom de alta resolução e transdutores de alta frequência (Graham 2008, Mogicato et al. 2011).

As mensurações obtidas pela ultrassonografia são indicadores confiáveis em casos de hiperplasia adrenal (Barthez et al. 1998, Baroni et al. 2002). Ressalta-se que o método possui função de triagem em casos de doença de Cushing, Addison ou neoplasias, podendo muitas vezes auxiliar no estabelecimento da origem da desordem endócrina (Zerbe 2000, Gould et al. 2001, Graham 2008).

As mensurações de referência e descrição de doenças nas glândulas adrenais são bem exploradas em espécies como cães e gatos (Barthez et al. 1998, Watson \& Herrtage 1998, Gould et al. 2001, Mogicato et al. 2011) e até mesmo em ferrets (Barthez et al. 1998, Besso et al. 2000). Todavia, poucos estudos ultrassonográficos descrevem-se as mensurações de referência das glândulas adrenais em primatas não humanos (Wagner \& Kirberger 2005, Amory et al. 2013). Assim como nota-se a falta de dados dos padrões de normalidade, verificam-se também poucas descrições sobre distúrbios das glândulas adrenais em pri- matas não humanos, apesar disso foram já detectados processos neoplásicos e hiperplasias, associadas ou não a sinais clínicos de hiperadrenocorticismo em algumas espécies (Cianciolo et al. 2007, Brown et al. 2009, David et al. 2009, Guardado-Mendoza et al. 2009, Jurczynskit et al. 2012).

Portanto, considerando a escassa literatura, questiona-se se as doenças nas glândulas adrenais em primatas não humanos são incomuns ou subdiagnosticadas, havendo a hipótese desse fato ser determinado pela falta de parâmetros.

Objetivou-se neste estudo descrever as características ultrassonográficas das glândulas adrenais para três espécies de primatas não humanos mantidas em cativeiro: Saimiri sciureus (mico-de-cheiro), Aotus azarae infulatus (macaco-da-noite) e Alouatta guariba clamitans (bugio-ruivo).

\section{MATERIAL E MÉTODOS}

Foram realizados exames ultrassonográficos abdominais em machos e fêmeas de três espécies de primatas não humanos neotropicais: Saimiri sciureus (mico-de-cheiro), Aotus azarae infulatus (macaco-da-noite) e Alouatta guariba clamitans (bugio-ruivo). Todos os animais avaliados eram provenientes de cativeiros, inteiros, sexualmente maduros e estavam em idade adulta. Informações sobre idade, peso e perímetro abdominal foram registradas para cada espécie (Quadro 1). A medida do perímetro abdominal foi realizada na altura da cicatriz umbilical, por meio de uma fita métrica.

As avaliações clínicas, exames de sangue tais como hemograma, perfil bioquímico hepático (ALT - alanina transaminase, AST aspartato transaminase, FA - fosfatase alcalina e GGT - gama glutamil transpeptidase), perfil bioquímico renal (creatinina e ureia), proteína total e número de plaquetas foram avaliados juntamente com o histórico clínico, objetivando avaliar a higidez dos animais.

Utilizou-se para os exames ultrassonográficos o equipamento da marca ESAOTE, Genova, Itália, modelo MyLab 30 Vet Gold, de modo bidimensional, com transdutor linear (LA435) de $10 \mathrm{MHz}$. Gel acústico foi utilizado para formação das imagens.

0 estudo foi dividido em duas partes distintas:

\section{Parte 1}

A primeira parte da coleta de dados foi realizada no Centro Nacional de Primatas (Cenp), localizado na rodovia BR-316, 7 km, na cidade de Ananindeua, região metropolitana de Belém, estado do Pará. Foi criado a partir da Portaria do Ministério da Saúde no Bsb-115 de 15 de março de 1978. É uma instituição federal subordinada ao Ministério da Saúde, fazendo parte da Secretaria de Vigilância em Saúde (SVS).

Foram submetidos a exames ultrassonográficos 24 animais da espécie Saimiri sciureus (mico-de-cheiro), 11 fêmeas e 13 ma-

Quadro 1. Relação dos animais avaliados ultrassonograficamente, discriminados por espécie, sexo, médias da idade, peso e perímetro abdominal

\begin{tabular}{lcccc}
\hline Espécie & Sexo & $\begin{array}{c}\text { Idade } \\
\text { (anos) }\end{array}$ & $\begin{array}{c}\text { Peso } \\
\text { (kg) }\end{array}$ & $\begin{array}{c}\text { Perímetro abdo- } \\
\text { minal }(\mathrm{cm})\end{array}$ \\
\hline Mico-de-cheiro & Fêmeas $(\mathrm{n}=11)$ & 7 & 0,644 & 3,07 \\
(n=24) & Machos $(\mathrm{n}=13)$ & 6 & 0,851 & 5,56 \\
Macaco-da-noite & Fêmeas $(\mathrm{n}=12)$ & 8 & 1,005 & 7,0 \\
(n=24) & Machos $(\mathrm{n}=12)$ & 11 & 1,085 & 8,6 \\
Bugio-ruivo & Fêmeas $(\mathrm{n}=10)$ & 7 & 3,87 & 29,4 \\
(n=22) & Machos $(\mathrm{n}=12)$ & 8 & 6,027 & 35,5
\end{tabular}


chos, com idade entre três e 13 anos e 24 animais da espécie Aotus azarae infulatus (macaco-da-noite), divididos entre 12 fêmeas e 12 machos, com idade entre quatro e 12 anos (Quadro 1). Tais animais são mantidos em cativeiro como modelos experimentais para diversas pesquisas, principalmente com febre amarela e malária.

Os exemplares de Saimiri sciureus habitam um galpão destinado às espécies poligâmicas, com 24 recintos de 2,30 x 3,85 x 2,55 metros (largura x comprimento $x$ altura), o qual possui quatro poleiros transversais e plataforma de ripas de madeira, conjugadas em duas partes, possuindo uma portinhola central que dá passagem aos animais. Tal disposição facilita o manejo e possibilita maior segurança aos tratadores. Cada conjunto de recinto duplo abriga um grupo reprodutivo de espécie poligâmica Saimiri sciureus, em média com um macho adulto e 10 fêmeas.

Os exemplares de Aotus azarae infulatus apresentam características próprias de biologia e requerem um manejo específico, portanto são instalados em um galpão destinado a espécies monogâmicas, o qual possui 48 recintos, dispostos em duas baterias de 24 , medindo $1,20 \times 3,85$ x 2,40m de altura (largura x comprimento $\mathrm{x}$ altura). No interior dos recintos há quatro poleiros transversais de $5 \mathrm{~cm}$ de diâmetro e plataformas de madeira nas laterais e no fundo do recinto em três níveis para viabilizar o banho de sol. Há também uma caixa ninho de metal e piso telado, fixada na parte frontal para abrigo e contenção. A alimentação é oferecida em bandeja de inox, fixada na parte frontal do recinto, sendo a água e leite oferecidos em mamadeiras de meio litro.

\section{Parte 2}

A segunda parte da coleta de dados foi realizada no Centro de Pesquisas Biológicas de Indaial e Observatório de Primatas Morro Geisler - Projeto Bugio, localizado na Rua Rio de Janeiro 1, Bairro dos Estados, Indaial, SC 89130-000. 0 Centro foi criado através da Lei Municipal no 2099, de 20 de março de 1992, e é mantido por meio de um convênio entre a Prefeitura Municipal de Indaial e a Fundação Universidade Regional de Blumenau (FURB).

Foram realizados exames ultrassonográficos em 22 exemplares de Alouatta guariba clamitans (bugio-ruivo), compostos por 12 machos e 10 fêmeas, com idade entre seis a 13 anos, mantidos em recintos após terem sidos resgatados nas proximidades apresentando na oportunidade algum tipo de enfermidade. No momento do exame tais animais apresentavam-se hígidos.

Os bugios encontravam-se instalados individualmente em recintos com dimensões de 3 x 5 x 2,6m (largura x comprimento $\mathrm{x}$ altura), com área coberta e fechada com dimensões de $3 \times 1,5$ x 2,6m, e enriquecidos com troncos, cordas, mangueiras e caixa com aquecimento. A dieta diária é composta de frutas, folhas e ração. As frutas e folhas são fornecidas três vezes ao dia, com horários semelhantes aos observados em ambiente natural, enquanto a ração apenas uma vez ao dia. A água é ofertada a vontade. Os cativeiros são higienizados diariamente e os animais monitorados periodicamente por Médico Veterinário.

Não foi realizado jejum prévio para espécies Saimiri sciureus e Aotus azarae infulatus devido às orientações dos tratadores quanto ao estresse dos animais, sendo retirados alimentos potencialmente fermentadores (frutas e folhas) e ofertada apenas ração. Para os exemplares de Alouatta guariba clamitans realizou-se jejum alimentar e hídrico prévio de 4 a 12 horas, porém alguns exemplares receberam folhas antes do exame.

As avaliações ultrassonográficas foram realizadas após a tricotomia do abdome ventral, entre o processo xifoide e a região inguinal, somente com contenção física, sem o uso de sedativos. Os animais foram mantidos em decúbito dorsal por 15 a $30 \mathrm{mi}-$ nutos. Objetivando minimizar o estresse dos animais foi ofertado leite condensado durante a avaliação dos macacos-da-noite, gli- cose para os bugios-ruivo e a visão foi obliterada com algum tipo de tecido durante o exame dos micos-de-cheiro, manobras essas indicadas pela equipe dos cativeiros.

0 exame foi realizado de forma completa, iniciando-se pelo fígado, em sentido horário, avaliando todos os órgãos da cavidade abdominal, também como meio de comprovação da higidez dos animais. Em sequência, objetivou-se a análise das janelas ultrassonográficas para visualização das glândulas adrenais e quando identificadas, suas características ultrassonográficas foram detalhadas nos diferentes cortes ultrassonográficos. Caracterizou-se a glândula quanto à forma (oval, semilunar ou bilobada), ecogenicidade (cortical e medular) e mensurações, tais como o comprimento (extremidade cranial a caudal) e a largura, considerando que mensuração da largura foi realizada sempre no polo maior e ao corte sagital. Foram realizadas duas medidas de cada glândula e calculadas as médias dos valores.

Além de análise estatística descritiva, os dados foram avaliados pelo programa estatístico utilizando o software JMP, versão 5.0.1 (Software JMP, versão 5.0.1, SAS Institute Inc., Cary, NC). As mensurações dos dados contínuos numéricos (comprimentos e larguras) entre adrenais esquerda e direita, bem como entre os sexos, foram analisados pelo teste t-pareado. Correlação quanto ao tamanho das adrenais e a variação de peso e perímetro abdominal dos animais foram avaliados pelo teste de correlação Bartlett (para comparar correlações das variáveis contínuas numéricas). Análises de variância (ANOVA) e Teste Exato de Fisher foram utilizados para comparações das medidas entre as espécies. Os dados foram expressos na forma de média \pm desvio padrão e valores de $\mathrm{P}<0,05$ foram considerados estatisticamente significativos.

Este estudo foi aprovado pelo Comitê de Ética no Uso de Animais do Setor de Ciências Agrárias da Universidade Federal do Paraná sob o número de protocolo 014/2011 em 29 de junho de 2011 e pelo Comitê de Ética ao Uso de Animais da Universidade Regional de Blumenau sob número 010/11 em 19 de outubro de 2011.

\section{RESULTADOS}

Após a análise dos exames ultrassonográficos, sangue, avaliação e histórico clínico, dois exemplares (um macho e uma fêmea) do grupo dos bugios (Alouatta guariba clamitans) foram excluídos do estudo. No grupo dos macacos-da-noite (Aotus azarae infulatus) notou-se que todos apresentavam, em menor ou maior intensidade, lesões hepáticas detectadas pela ultrassonografia ou pelo perfil bioquímico hepático, provavelmente como consequência de experimentos anteriores. Apesar disso, esses animais não apresentavam sinais clínicos relacionados a doenças nas glândulas adrenais e foram incluídos no estudo.

Apenas um macaco-da-noite e quatro micos-de-cheiro apresentaram neutrofilia leve, porém em nenhum animal notou-se leucograma de estresse característico (neutrofilia, monocitose e linfopenia), sendo assim, nenhum animal dessas espécies foi excluído do estudo.

0 rim foi o principal órgão de referência em relação a ambas às glândulas adrenais. Notou-se em todas as espécies analisadas que a posição da visualização ultrassonográfica da adrenal esquerda é ínfero-medial em relação ao polo cranial do rim esquerdo, tendo como limite cranial o fígado ou estômago, limite caudal o rim, limite lateral as alças intestinais ou o baço e como limite medial a aorta. Para visualização desta glândula o transdutor deve ser posicionado longitudinalmente ao polo cranial do rim esquerdo e 
angulado em torno de $20^{\circ}$ a $30^{\circ}$ medialmente, em seguida a superfície do transdutor também deve ser rotacionada medialmente até a imagem renal desaparecer.

A glândula direita localiza-se mais cranialmente em relação à esquerda, em uma posição variando de supra-renal a ínfero-medial, tendo como limite cranial e lateral o fígado, limite caudal o polo cranial do rim direito e limite medial a veia cava. 0 transdutor deve ser posicionado longitudinalmente ao polo cranial direito e direcionado levemente em sentido medial. Em alguns casos é necessário rotacionar o transdutor até o rim desaparecer e em outros a imagem renal direita e adrenal são formadas em um mesmo plano.

As glândulas adrenais não foram identificadas em todos os animais, conforme pode ser observado no Quadro 2.

As glândulas adrenais apresentaram morfologia muito semelhante nas três espécies estudadas, apresentando característica oval a semilunar, variando entre alongado, arredondado ou tênue marcação da cintura entre os polos, sendo esta cintura raramente pronunciada em alguns casos (9/103), como demonstrado na Quadro 3 e Figuras 1 a 3.

A diferenciação da camada cortical e medular, bem como a junção pôde ser observada apenas em alguns animais (Quadro 3), sendo que a camada cortical mostrou-se hipoecóica e a medular hiperecóica.

As mensurações foram estabelecidas em todas as glândulas visualizadas, as quais estão listadas na Quadro 4.

0 Teste Exato de Fisher mostrou que não houve significância estatística entre as mensurações de comprimento e

Quadro 2. Percentagem das avaliações ultrassonográficas das glândulas adrenais nas diferentes espécies de primatas não humanos, de acordo com a visualização em cada espécie

\begin{tabular}{|c|c|c|c|c|}
\hline \multirow[t]{2}{*}{ Espécie } & \multicolumn{2}{|c|}{ Adrenal esquerda } & \multicolumn{2}{|c|}{ Adrenal direita } \\
\hline & Visualizadas & $\begin{array}{c}\text { Não } \\
\text { visualizadas }\end{array}$ & Visualizadas & $\begin{array}{c}\text { Não } \\
\text { visualizadas }\end{array}$ \\
\hline $\begin{array}{l}\text { Mico-de-cheiro } \\
(n=24)\end{array}$ & $50 \%$ & $50 \%$ & $62,5 \%$ & $37,5 \%$ \\
\hline $\begin{array}{l}\text { Macaco-da-noite } \\
(n=24)\end{array}$ & $87,5 \%$ & $12,5 \%$ & $95,8 \%$ & $4,2 \%$ \\
\hline $\begin{array}{l}\text { Bugio-ruivo } \\
(n=20)\end{array}$ & $85 \%$ & $15 \%$ & $75 \%$ & $25 \%$ \\
\hline
\end{tabular}

largura entre os animais da espécie Aotus azarae infulatus e Saimiri sciureus, todavia houve diferença entre o Alouatta guariba clamitans e as demais espécies.

Em relação às mensurações dos comprimentos e larguras das glândulas esquerda e direita, não houve variação estatística significante em nenhuma espécie.

Quanto ao gênero houve diferença estatística somente nos bugios para o comprimento e largura.

A análise de correlação das mensurações com o peso e o perímetro abdominal demonstrou correlação positiva entre o perímetro abdominal e a largura das adrenais nos micos-de-cheiro e correlação positiva entre o peso e comprimento das adrenais nos bugios.

Durante os cálculos de correlações das mensurações entre peso e perímetro abdominal nos macacos-da-noite (Aotus azarae infulatus) retirou-se um dos dados por ser detectado como discrepante, isto é, um "out lier" com base análises de quartis.

\section{DISCUSSÃO}

A avaliação das glândulas adrenais é dependente da utilização de um equipamento adequado com alta resolução e transdutores de alta frequência, fator considerado nesse estudo (Graham 2008, Mogicato et al. 2011).

A manobra de contenção física proposta por Christman (2010) foi satisfatória para um bom exame das glândulas adrenais. Considera-se que para primatas de pequeno porte $(<5 \mathrm{~kg})$ a contenção física realizada com equipamentos de proteção individual (luvas e máscaras) deve ser o método de escolha para realização de procedimentos que não causem dor e não ofereçam riscos de saúde tanto para o animal como para a equipe, apesar dos bugios machos ultrapassarem discretamente esse peso (Fowler 1995, Christman 2010). Todavia considera-se a dificuldade da imobilização dos animais muitos estressados, em todas as espécies avaliadas.

Optou-se por incluir na análise dos dados as mensurações das glândulas adrenais obtidas em macacos-da-noite que apresentaram alterações hepáticas e dos micos-de-cheiro com discretas variações no hemograma, para determinar melhor os grupos dos animais. Tal fato baseado

\section{Quadro 3. Características do aspecto e morfologia de glândulas adrenais avaliadas por meio de exame ultrassonográfico, discriminados em esquerda e direita e por espécie avaliada}

\begin{tabular}{|c|c|c|c|c|c|c|c|}
\hline \multirow{2}{*}{$\begin{array}{l}\text { Espécie } \\
\text { Mico-de-cheiro }\end{array}$} & \multirow{2}{*}{$\begin{array}{c}\text { Característica } \\
\mathrm{n}\end{array}$} & \multicolumn{3}{|c|}{ Adrenal esquerda } & \multicolumn{3}{|c|}{ Adrenal direita } \\
\hline & & & 12 & & \multirow{4}{*}{$\begin{array}{c}\text { Oval } \\
93,3 \%\end{array}$} & \multicolumn{2}{|l|}{12} \\
\hline & \multirow[t]{2}{*}{ Aspecto } & Oval & Bilobado & \multirow{3}{*}{$\begin{array}{c}\text { Semilunar } \\
0 \%\end{array}$} & & Bilobado & Semilunar \\
\hline & & $75 \%$ & $25 \%$ & & & $0,7 \%$ & $0 \%$ \\
\hline & $\begin{array}{c}\text { Definição } \\
\text { corticomedular }\end{array}$ & $33 \%$ & $26,6 \%$ & & & & \\
\hline \multirow[t]{5}{*}{ Macaco-da-noite } & $\mathrm{n}$ & & 21 & & & 23 & \\
\hline & Aspecto & Oval & Bilobado & Semilunar & Oval & Bilobado & Semilunar \\
\hline & & $61,9 \%$ & $0 \%$ & $38,1 \%$ & $21,7 \%$ & $13,1 \%$ & $65,2 \%$ \\
\hline & $\begin{array}{c}\text { Definição } \\
\text { corticomedular }\end{array}$ & $47,6 \%$ & $39,1 \%$ & & & & \\
\hline & $\mathrm{n}$ & & 17 & & & 15 & \\
\hline \multirow[t]{2}{*}{ Bugio-ruivo } & Aspecto & Oval & Bilobado & Semilunar & Oval & Bilobado & Semilunar \\
\hline & $\begin{array}{c}\text { Definição } \\
\text { corticomedular }\end{array}$ & $23,5 \%$ & $33,3 \%$ & & 870 & & \\
\hline
\end{tabular}




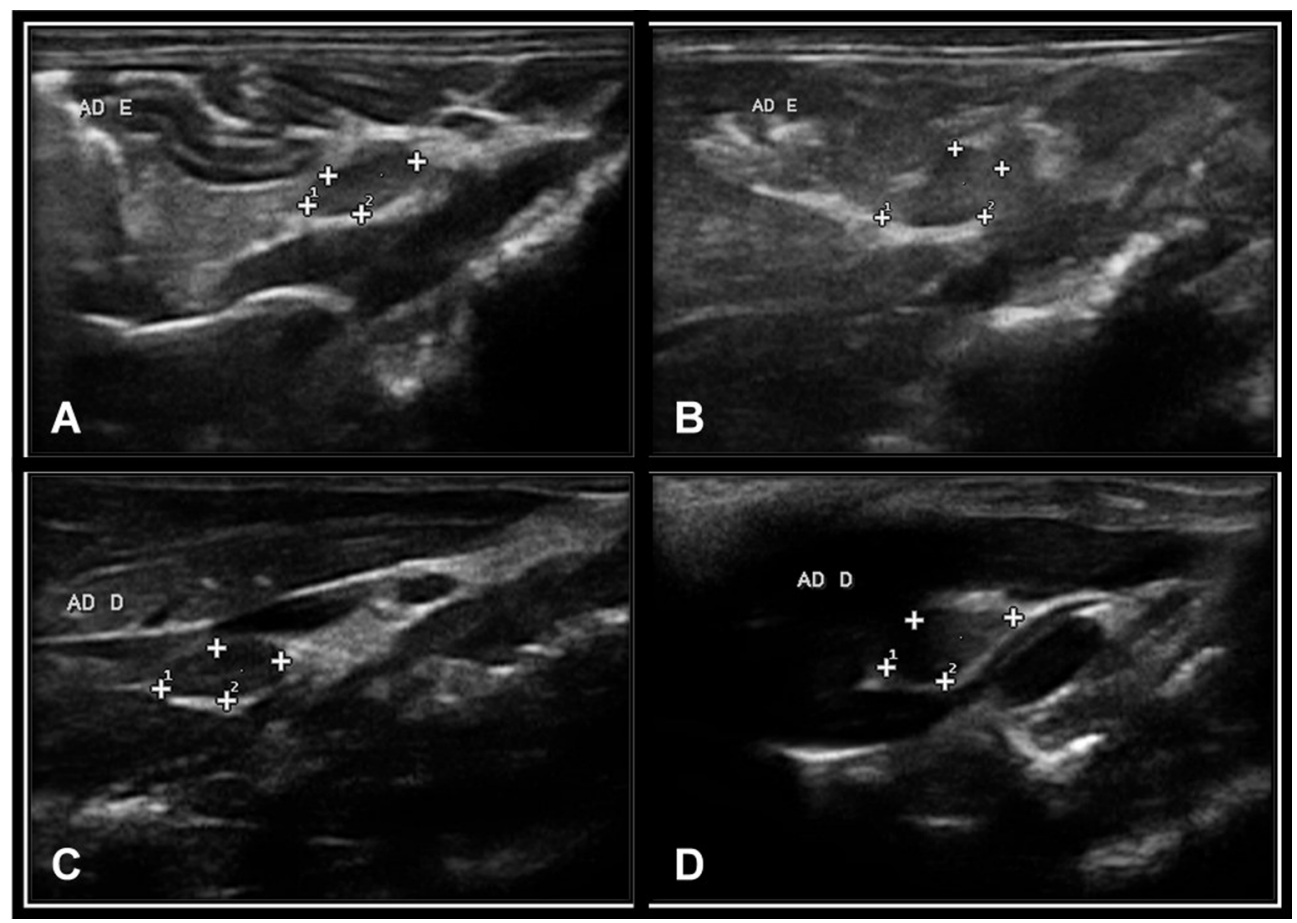

Fig.1. Imagens ultrassonográficas obtidas com transdutor linear de $10 \mathrm{MHz}$ das glândulas adrenais de exemplar de Saimiri sciureus. (A) Glândula adrenal esquerda com aspecto oval, sem diferenciação corticomedular. (B) Glândula adrenal esquerda com aspecto bilobado, sem diferenciação corticomedular. (C) Glândula adrenal direita com aspecto oval e diferenciação corticomedular. (D) Glândula adrenal direita com aspecto bilobado e sem diferenciação corticomedular.

em estudo recente (Mogicato et al. 2011) no qual descreve que não houve diferença estatística nas mensurações das adrenais entre animais saudáveis e não saudáveis.

A posição da adrenal esquerda (ínfero-medial ao rim esquerdo) e direita (variando de cranial a ínfero-medial ao rim direito) corroboram com dados encontrados por Wagner \& Kirberger (2005), Amory et al. (2013) e se assemelham aos seres humanos (Baroni et al. 2002).

A formação da imagem da glândula esquerda proporcionou maior dificuldade em comparação à glândula direita nas três espécies de primatas não humanos avaliados (Quadro 2), fato esse também descrito em seres humanos por Baroni et al.(2002), ao contrário do relatado em pequenos animais (Barthez et al., 1998, Mogicato et al. 2011). Acredita-se que o principal motivo foi na dificuldade de obtenção da imagem da adrenal esquerda foi em decorrência a topografia do baço, o qual se localiza adjacente à adrenal esquerda e eventualmente cortes de porções desse órgão aparecem no mesmo plano da glândula, confundindo o ultrassonografista, por ecogenicidades semelhantes (Wagner \& Kirberger 2005).

Além disso, outro fator prejudicial para obtenção de boa janela acústica para a adrenal esquerda foi a distensão do trato gastrintestinal, presente em todas as espécies, porém mais evidenciado nos bugios. Alguns exemplares apresentaram artefatos gerados pelo acúmulo de alimento e gás no estômago e nas alças do intestino, o que impossibilitou a identificação das glândulas, enquanto que a captação da imagem foi facilitada para os animais com estômago con- traído. A não cooperação, inquietação e taquipneia decorrente do estresse da contenção, prejudicaram a avaliação e foram observadas em todas as espécies, aspectos também pronunciados nos bugios, provavelmente pelo porte ser maior em relação às demais espécies, motivos esses já percebidos em cães, gatos e ferrets (Schelling 1991).

Embora tenha sido mais fácil visibilizar a adrenal direita na maioria das espécies, o maior número de visualizações de adrenais esquerdas em relação às direitas nos bugios foi decorrente do maior estresse e exaustão observados nessa espécie, já que a adrenal direita era último órgão a ser avaliado na cavidade abdominal. Devido à preocupação com a integridade física do animal, optou-se por não realizar outras tentativas.

Apesar do contraste entre a ecogenicidade da glândula adrenal e da gordura abdominal adjacente, as avaliações realizadas em animais magros de exemplares de macacos-da-noite e bugios-ruivo foram melhores em relação aos animais em sobrepeso, isto é, o pouco acúmulo de gordura intra-abdominal mostrou-se como facilitador do exame. Em contrapartida, o porte do animal e menor perímetro abdominal (o menor em comparação), característicos dos micos-de-cheiro, foram os principais complicadores dessa análise, sendo a principal causa da não diferenciação das glândulas nessa espécie. Destaca-se ainda a adaptação do observador às espécies, notando-se que nos primeiros animais examinados da espécie Saimiri sciureus (mico-de-cheiro) e Alouatta guariba clamitans (bugio-ruivo) não foram identificadas as glândulas adrenais. 

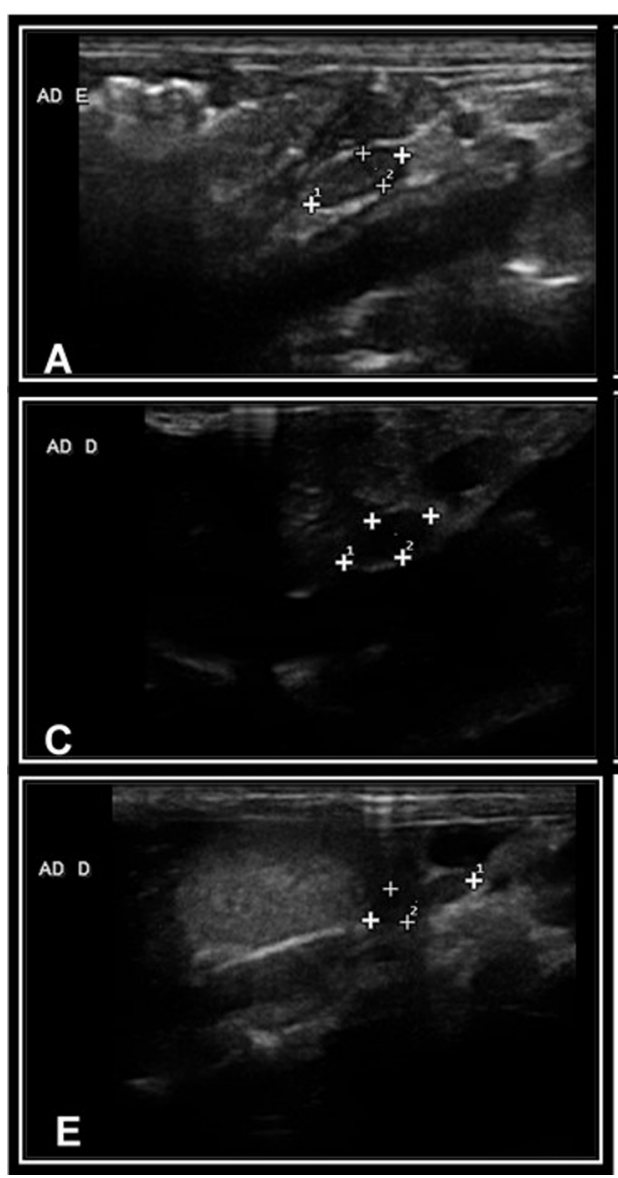

\section{D}
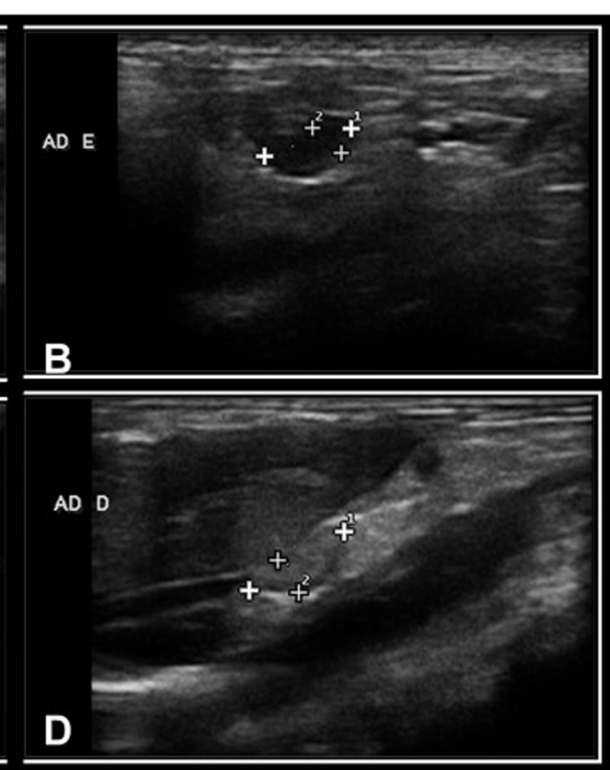

Fig.2. Imagens ultrassonográficas obtidas com transdutor linear de $10 \mathrm{MHz}$ das glândulas adrenais de um exemplar de Aotus azarae infulatus. (A) Glândula adrenal esquerda com aspecto oval e diferenciação corticomedular. (B) Glândula adrenal esquerda com aspecto semilunar e sem diferenciação corticomedular. (C) Glândula adrenal direita com aspecto oval, sem diferenciação corticomedular. (D) Glândula adrenal direita com aspecto semilunar e diferenciação corticomedular. (E) Glândula adrenal direita com aspecto bilobado, sem diferenciação corticomedular.
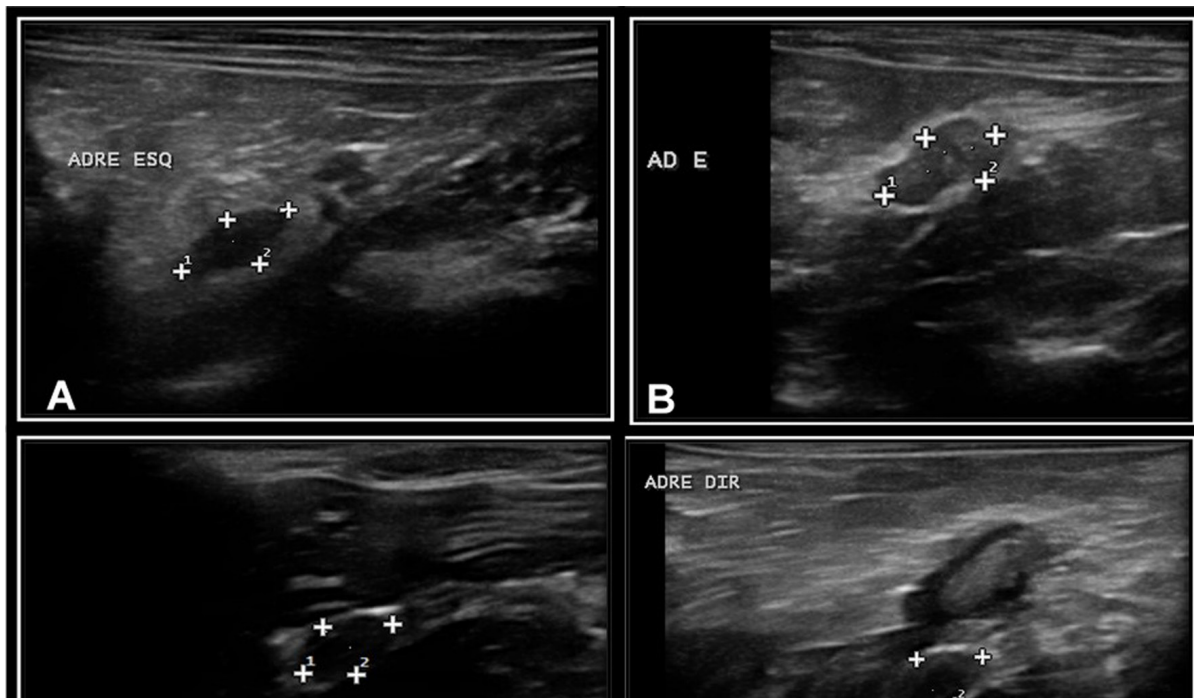

ADREN DIR

C

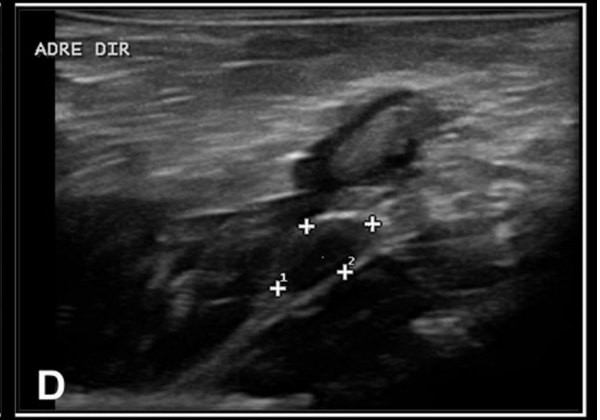

Fig.3. Imagens ultrassonográficas obtidas com transdutor linear de $10 \mathrm{MHz}$ das glândulas adrenais de um exemplar de Alouatta clamitans. (A) Glândula adrenal esquerda com aspecto oval, sem diferenciação corticomedular. (B) Glândula adrenal esquerda com aspecto bilobado e tênue diferenciação corticomedular. (C) Glândula adrenal direita com aspecto oval, sem diferenciação corticomedular. (D) Glândula adrenal direita com aspecto semilunar e diferenciação corticomedular. 
Quadro 4. Medidas de comprimento e largura das glândulas adrenais, esquerda e direita, mensuradas por meio de ultrassonografia abdominal em diferentes espécies de primatas não humanos, discriminadas por espécie

\begin{tabular}{|c|c|c|c|c|}
\hline \multirow[t]{2}{*}{ Espécie } & \multicolumn{2}{|c|}{ Adrenal esquerda } & \multicolumn{2}{|c|}{ Adrenal direita } \\
\hline & $\begin{array}{l}\text { Comprimento } \\
\text { (mm) }\end{array}$ & $\begin{array}{c}\text { Largura } \\
(\mathrm{mm})\end{array}$ & $\begin{array}{c}\text { Comprimento } \\
(\mathrm{mm})\end{array}$ & $\begin{array}{c}\text { Largura } \\
(\mathrm{mm})\end{array}$ \\
\hline Mico-de-cheiro & $7,0 \pm 0,47^{a *}$ & $3,23 \pm 0,54^{\mathrm{a}}$ & $7,33 \pm 1,02^{a}$ & $3,68 \pm 0,58^{a}$ \\
\hline Macaco-da-noite & $7,26 \pm 1,15^{a}$ & $3,42 \pm 0,54^{\mathrm{a}}$ & $7,65 \pm 1,28^{\text {a }}$ & $3,75 \pm 0,83^{\text {a }}$ \\
\hline Bugio-ruivo & $9,9 \pm 1,76^{b *}$ & $4,96 \pm 1,17^{b}$ & $9,07 \pm 2,44^{b}$ & $5,43 \pm 0,95^{b}$ \\
\hline
\end{tabular}

A dificuldade em distinguir as glândulas e grandes vasos não ocorreu durante as avaliações neste estudo, assim como percebido por Wagner \& Kirberger (2005), sendo este fato citado em cães e gatos (Barthez et al. 1998). Todavia, isso ocorreu com os linfonodos mesentéricos, os quais necessitavam ser isolados cuidadosamente da glândula adrenal esquerda, principalmente pela sua posição e ecogenicidade, considerando que seu aspecto apresentava-se mais alongado.

As características morfológicas das glândulas avaliadas, principalmente em relação à forma (Quadro 3), podem ter sofrido interferência da janela acústica utilizada na captação da imagem, considerando que os animais movimentavam-se diversas vezes durante o exame. Apesar de terem sido descritas morfologias variadas, o aspecto oval e bilobado prevaleceram paras as glândulas adrenais esquerdas, assim como descrito por Amory et al. (2013). Já para a glândula direita não foi possível determinar uma forma predominante, essa apresentando em aspecto oval e semilunar, porém não em formato em "V", como sugerido por Amory et al. (2013) em macaco verde, outro tipo de primata não humano.

Interessante ressaltar que essas diferenças entre as formas não são evidenciadas em cães e gatos, que apresentam caracteristicamente a adrenal esquerda bilobada e a direita com aspecto oval alongado (Graham, 2008). Notou-se, portanto, que primatas não humanos apresentam características singulares, não sendo possíveis comparações com cães e gatos ou seres humanos (Baroni et al. 2002).

A diferenciação entre a camada cortical e a medular, bem como a identificação da junção corticomedular foi possível em 35 das 103 glândulas avaliadas (Quadro 3), similar ao descrito em outros primatas (Wagner \& Kirberger 2005, Amory et al. 2013) e em pequenos animais (Graham, 2008). Todavia notou-se baixa percentagem da identificação dessa característica em relação a estudos recentes em cães (Mogicato et al. 2011), fato que pode ser explicado provavelmente pela dificuldade de janela acústica nos primatas como supracitado. 0 aspecto hipoecóico da cortical e hiperecóico da medular detectado também é uma característica comum aos pequenos animais (Barthez et al. 1998, Graham 2008).

As análises dos dados revelaram uma diferença estatística para o comprimento e a largura entre os gêneros nos bugios-ruivo (Quadro 5), o que pode ser explicado pelo dimorfismo sexual evidente nessa espécie (Verona \& Pissinatti 2006). Os machos apresentam peso e perímetro abdo- minal maiores em relação às fêmeas, fato comprovado nesse estudo e por demais autores (Verona \& Pissinatti 2006). A correlação positiva detectada nos bugios-ruivo, entre comprimento das adrenais e peso, corrobora com dados descritos para cães (Mogicato et al. 2011). Já a correlação entre largura das adrenais e perímetro abdominal, encontrada nos micos-de-cheiro, foram descritas pela primeira vez por este estudo.

O Teste Exato de Fisher revelou que não houve diferença estatística entre os macacos-da-noite e os micos-de-cheiro, provavelmente devido à similaridade do porte desses animais. Seguindo esse raciocínio, a significância estatística demonstrada entre os bugios-ruivo as outras duas espécies, provavelmente seja em consequência do maior porte observado nesses primatas.

Sabe-se que a adaptação de animais em ambientes muitos estressantes, como cativeiros, é frequentemente acompanhada por uma hipertrofia das glândulas adrenais, predominantemente do córtex, responsável pela liberação de hormônios corticosteroides, ligados intimamente com o estresse crônico, como o cortisol (Cunningham 2004). Portanto deve-se levar em consideração que apesar de não haver sinais clínicos com relação a distúrbios nas adrenais, as mensurações realizadas nesse estudo foram estabelecidas em animais de cativeiro.

\section{CONCLUSÕES}

Conclui-se que é possível a identificação das glândulas adrenais por meio de exame ultrassonográfico nas espécies de primatas não humanos avaliadas, bem como a realização de mensurações.

Os padrões de referência foram estabelecidos com sucesso para as espécies em questão, sendo que os dados podem ser extrapolados para outras espécies similares, em que o porte, peso e perímetro abdominal forem semelhantes.

Ressalta-se que apesar de apresentarem particularidades, a posição e o aspecto das glândulas adrenais devem ser comparados aos dos seres humanos preferencialmente aos animais domésticos.

\section{REFERÊNCIAS}

Amory J.T., Du Plessis W.M., Beierschmitt A., Beeler-Marfisi J., Palmour R.M. \& Beths T. 2013. Abdominal ultrasonography of the normal St. Kitts vervet monkey (Chlorocebus sabaeus). J. Med. Primatol. 42:28-38.

Baroni R.H., Angare C.G. \& Sjenzfeld J. 2002. Retroperitônio e Adrenais, p.470-489. In: Cerri G.G. \& Oliveira I.R.S. (Eds), Ultra-Sonografia Abdominal. Revinter, Rio de Janeiro.

Barthez P.Y., Nyland T.G. \& Feldman E.C. 1998. Ultrasonography of the adrenal glands in the dog, cat, and ferret. Vet. Clin. North Am., Small Anim. Pract. 28:869-885.

Besso J.G., Tidwell A.S. \& Gliatto J.M. 2000. Retrospective review of the ultrasonography features of adrenal lesions in 21 ferrets. Vet. Radioll. Ultrasound 41:345-352.

Brown S.L., Anderson D.C., Dick J.R., Guardado-Mendonza R., Garcia A.P. \& Hubbard G.B. 2009. Neoplasia in the chimpanzee (Pan spp.). J. Med. Primatol. 38:137-144.

Christman J. 2010. Physical methods of capture, handling, and restraint of mammals, p.39-48. In: Kleiman D.G., Thompson K.V. \& Baer C.K. (Eds), Wild Mammals in Captivity: principles and techniques for zoo management. $2^{\text {nd }}$ ed. Chicago Press, Chicago. 
Cianciolo R.E., Butler S.D., Eggers J.S., Dick Jr E.J., Leland M.M., Garza M., Brazky K.M., Cummins L.B. \& Hubbard G.B. 2007. Spontaneous neoplasia in the baboon (Papio spp.). J. Med. Primatol. 36:61-79.

Cunningham J.G. 2004. Tratado de Fisiologia Veterinária. Guanabara Koogan, Rio de Janeiro, p.357-368.

David M.J., Dick Jr E.J. \& Hubbard G.B. 2009. Spontaneous pathology of the common marmoset (Callithrix jacchus) and tamarins (Saguinus oedipus, Saguinus mystax). J. Med. Primatol. 38:347-359.

Fowler M.E. 1995. Restraint and Handling of Wild and Domestic Animals. Iowa State University, Ames, p.236-246.

Gould S.M., Baines E.A., Mannion P.A., Evans H. \& Herrtage M.E. 2001. Use of endogenous ACTH concentration and adrenal ultrasonography to distinguish the cause of canine hyperadrenocorticism. J. Small Anim. Pract. 42:113-121.

Graham J. 2008. Adrenal glands, p.383-394. In: Penninck D. \& D’Anjou M.A. (Eds), Atlas of Small Animal Ultrasonography. Blackwell Publishing, Iowa.

Jurczynski K., Gruber-Dujardin E., Widmer D.E., Kaup F.J. \& Mätz-Rensing K. 2012. Invasive aspergillosis in a Putty-nosed monkey (Cercopithecus nictitans) with adrenocortical Cushing's Syndrome. J. Med. Primatol. 41:172-175.

Mogicato G., Layssol-Lamour C., Conchou F., Diquelou A., Raharison F., Sautet J. \& Concordet D. 2011. Ultrasonographic evaluation of the adrenal glands in healthy dogs: repeatability, reproducibility, observer-dependent variability, and the effect of bodyweight, age and sex. Vet Rec. 168:130.

Schelling C.G. 1991. Ultrasonography of the adrenal gland. Probl. Vet. Med. 3:604.

Verona C.E.S. \& Pissinatti A. 2006. Primates - Primatas do Novo Mundo (Sagui, Macaco-prego, Macaco-aranha, Bugio), p.358-377. In: Cubas Z.S., Silva J.C.R. \& Catão-Dias J.L. (Eds), Tratado de Animais Selvagens: medicina veterinária. Roca, São Paulo,

Wagner W.M. \& Kirberger R.M. 2005. Transcutaneous ultrasonography of the abdomen in the normal common marmoset (Callithrix jacchus). Vet. Radiol. Ultrasound 46:251-258.

Watson P.J. \& Herrtage M.E. 1998. Hyperadrenocorticism in six cats. J. Small Anim. Pract. 39:175-184.

Zerbe C.A. 2000. Differentiating tests to evaluate hyperadrenocorticism in dogs and cats. Comp. Contin. Educ. Pract. Vet. 22:149-157. 